\title{
Criminologie
}

\section{Des tâches policières privatisées à une police grise : quatre recherches belges en la matière}

\section{Lode Van Outrive}

Volume 31, numéro 2, automne 1998

La sécurité privée

URI : https://id.erudit.org/iderudit/017416ar

DOI : https://doi.org/10.7202/017416ar

Aller au sommaire du numéro

Éditeur(s)

Les Presses de l'Université de Montréal

ISSN

0316-0041 (imprimé)

1492-1367 (numérique)

Découvrir la revue

Citer cet article

Van Outrive, L. (1998). Des tâches policières privatisées à une police grise : quatre recherches belges en la matière. Criminologie, 31(2), 7-30.

https://doi.org/10.7202/017416ar
Résumé de l'article

This article summarizes the combined results of four branches of research concerning the privatization of policing tasks and the interaction between private agencies and police conducted between 1988 and 1997 in Belgium. The first segment focused on private detectives. A second research segment converged on the evolution of specialized investigations in insurance fraud. A third branch was aimed at the lack of legal protection available to citizens that had been subject to private investigations. The final segment consisted of an analysis of " grey policing". All research segments developed from a certain number of hypothesis and were based exclusively on qualitative research methods. Results from each segment have been assessed alongside established theories. 
DES TÂCHES POLICIÈRES PRIVATISÉES À UNE POLICE GRISE : QUATRE RECHERCHES BELGES EN LA MATIĖRE

Lode Van Outrive ${ }^{1}$

This article summarizes the combined results of four branches of research concerning the privatization of policing tasks and the interaction between private agencies and police conducted between 1988 and 1997 in Belgium. The first segment focused on private detectives. A second research segment converged on the evolution of specialized investigations in insurance fraud. A third branch was aimed at the lack of legal protection available to citizens that had been subject to private investigations. The final segment consisted of an analysis of "grey policing". All research segments developed from a certain number of hypothesis and were based exclusively on qualitative research methods. Results from each segment have been assessed alongside established theories.

\section{ÉLÉMENTS DE CONTEXTE}

Avec l'équipe de recherche Organisation policière et judiciaire du département de droit pénal, procédure pénale et criminologie, de l'université de Leuven, en Belgique, nous avons entrepris, entre 1988 et 1996, quatre recherches dans le domaine des tâches policières privatisées, secteur privé de la sécurité ${ }^{2}$. Ces recherches s'inscrivent dans un contexte particulier que nous exposerons d'abord.

En Belgique, comme dans d'autres pays occidentaux, le secteur de la sécurité privée a connu une expansion assez considérable à la fin des années 1970 et au début des années 1980 , période de crise fiscale, d'accroissement du chômage, de conflits sociaux mais aussi de grand banditisme. Au cours de cette période, un gouvernement centre droite a favorisé la flexibilité et la

1. Professeur émérite, U.K. de Leuven, Hooverplein 10, B-3000, Leuven, Belgique.

2. «Le syntagme recouvre un ensemble de travailleurs indépendants, d'entreprises ou d'organisations qui fournissent des services se rapportant à la défense des biens, des personnes et de l'information, soit à une clientèle spécifique contre rémunération, soit à l'individu ou à l'entité qui les emploie, soit à eux-mêmes, afin de protéger leur personne contre différentes menaces, risques techniques ou malveillance humaine » (Ocqueteau, 1990: 97). Cette définition couvre toutes nos populations de recherche, sauf les stewards qui sont censés collaborer à maintenir l'ordre dans les stades de football... mais qui, selon nous, fonctionnent en fait illégalement comme des agents privés de gardiennage et de surveillance. 
déréglementation du travail et entamé la privatisation d'institutions et de services publics. Ce même gouvernement a laissé libre cours au développement du secteur de gardiennage et de surveillance privés et a préparé, en cette matière, un projet de loi très libéral, ceci malgré le fait que les services de police, monopoles du maintien de l'ordre dans l'État latino-jacobin belge, avaient une image assez négative des gardiens privés (Van Outrive, 1988, 1990). La démission de ce gouvernement a eu pour effet de rendre ce projet de loi caduc.

En 1988, un nouveau gouvernement, cette fois centre-gauche, s'installe et manifeste la volonté de réglementer et de contrôler de façon plus adéquate les activités privées de surveillance, de gardiennage, mais aussi d'investigation. Ce nouveau projet devait dès lors remplacer les anciennes lois de 1933 et 1934 qui prohibaient la formation de milices privées uniformisées, tout en faisant des exceptions, sous certaines conditions, pour des compagnies de gardiennage et de surveillance. Avant de légiférer, le ministre des Affaires intérieures voulait cependant connaître l'envergure du phénomène, qu'il soit question de détectives privés classiques, d'agents spécialisés travaillant comme investigateurs privés contractuels ou d'employés spécialisés travaillant dans des compagnies privées comme les banques, les compagnies d'assurances ou les entreprises de distribution. À la demande du ministre, nous avons entrepris deux premières recherches portant sur ces populations. L'objet d'une troisième et d'une quatrième recherches fut ensuite laissé à notre entière initiative. Or, lors des deux premières recherches nous avions pu constater qu'il était indispensable de souligner l'importance de l'interaction entre la police publique et les agents (ou agences) privés, ce que nous nommions la police grise (grey policing). En outre, nous avions observé des phénomènes de non-respect des droits fondamentaux des citoyens dans les activités des agents privés. Ces deux situations allaient définir les troisième et quatrième recherches dont il sera question ici.

Après avoir présenté notre méthode de recherche - nous avons principalement travaillé en utilisant une méthode qualitative - nous exposerons la teneur de chacune des quatre recherches citées en rubrique ainsi que les hypothèses qui les ont initiées. Nous exposerons ensuite nos résultats en les mettant en rapport avec les meso-théories qui sont le résultat de précédentes recherches menées ailleurs, surtout aux États-Unis, aux Pays-Bas et en France. Nous sommes, en définitive, très conscient des aspects lacunaires de nos recherches et du terrain restant encore à explorer et à exploiter.

\section{L'USAGE DE LA MÉTHODE QUALITATIVE}

Nous avons combiné plusieurs techniques de recherche sans nous contraindre à respecter un quelconque ordre de succession des étapes. 
L'entretien non directif constitue notre principal mode de recueil de données. Ainsi, les chercheurs ont procédé à des interviews de personnes issues de différents milieux : policiers, hauts fonctionnaires de la justice, experts, détectives privés, informateurs, employés d'entreprises, magistrats et avocats. Soit ces personnes étaient impliquées dans l'une ou l'autre affaire, soit elles occupaient une position privilégiée nous permettant d'espérer l'obtention d'informations utiles à certains aspects de la recherche. L'utilisation de l'interview ouvert ne nous a pas empêchés de recourir à l'emploi d'un questionnaire fermé en fin de parcours afin de nous procurer des informations spécifiques. Nous faisons ici allusion à des questionnaires envoyés par courrier aux compagnies d'assurances belges à la fin de la seconde étude.

L'observation participante fut aussi employée, surtout dans la deuxième et la quatrième recherches. Par ailleurs, nous avons constamment fait usage de l'analyse de documents, tout en étant bien sûr tributaires de leur disponibilité. Ainsi, lors de la deuxième recherche, nous avons effectué une analyse de dossiers d'assurances. Alors que pour la quatrième, une étude approfondie de dossiers judiciaires et une analyse de la presse furent réalisées.

Nous avons également eu recours à la triangulation, pas nécessairement dans le but de valider nos découvertes ou de comparer des informations émanant de différentes sources, mais plutôt avec le souci d'obtenir des informations complémentaires. Nous concédons qu'une telle application de la triangulation peut parfois avoir été utilisée pour compenser un manque de temps.

Nous n'avons pas cherché à être représentatifs, au sens traditionnel du terme. Notre but étant de détecter un maximum de phénomènes, de modalités et de mécanismes différents, nous nous sommes plutôt appuyés sur le principe de saturation, c'est-à-dire que nous avons clôturé nos entretiens, observations ou analyses de documents dès que nous ne décelions plus d'informations nouvelles. Nous devons admettre que le laps de temps relativement limité dont nous disposions pour mener chaque recherche - en principe deux ans - représentait un obstacle qui nous a parfois forcés à mettre prématurément un terme à nos enquêtes. Ainsi, nos échantillons ne furent jamais représentatifs, mais rationnels (Albarello, Digneffe, Hiemaux, Maroy, Ruquoy et de Saint Georges, 1995). Qu'il s'agisse d'un échantillonnage de matériau documentaire ou d'une population d'acteurs, nous avons utilisé des critères multiples afin d'atteindre une certaine rationalité échantillonnale. Tout d'abord, un critère de complétude : nous avons retenu, dans les témoignages auxquels nous avions accès, ceux comportant le plus d'informations possibles. Ensuite, un critère de diversité interne : nous avons accordé une priorité à la variété et à la richesse des interactions entre les divers acteurs. Enfin, un critère de contraste et de diversité externe : nous avons essayé de donner 
une vision d'ensemble des différents types de situations auxquelles on pouvait se trouver confronté. Le critère majeur d'échantillonnage a finalement été le principe de diversification externe et interne, renvoyant au modèle d'échantillon par contraste (Pires, 1997). En effet, notre but était d'ouvrir la voie à une sorte de totalité hétérogène nous permettant de reconstruire une sorte de maquette d'interactions portant sur notre objet d'étude (Pires, 1997). Puisque, de prime abord, les populations concernées ne nous étaient pas familières, nous avons dû employer la technique dite boule de neige, technique pour le moins difficile à manier lorsqu'on veut éviter l'écueil qui consiste à sélectionner des échantillons d'un même groupe ou d'une même catégorie de personnes.

En termes d'analyse, il nous est souvent arrivé de ne pas pouvoir dépasser le stade descriptif et nous n'avons pas toujours pu effectuer des analyses structurelles au plan macro ou même meso. Notre meilleur exemple d'analyse de type meso-explication se rapporte à l'étude portant sur la police grise (Decorte et Van Laethem, 1997 ; Decorte, Van Laethem et Van Outrive, 1997). L'analyse la plus descriptive fut celle associée à la première étude portant sur les enquêteurs privés polyvalents.

\section{QUATRE RECHERCHES EMPIRIQUES}

\section{Les détectives privés de la première génération}

En 1988, nous avons donc entamé un projet de recherche à la demande du ministère des Affaires intérieures. Cette recherche portait sur l'état actuel des activités des détectives ou investigateurs privés (Cappelle, 1990). Une étude du marché belge de ces agents privés a révélé que quelque 139 détectives privés s'identifient comme tel. La plupart d'entre eux sont des indépendants. Certains fuient la publicité et sont, par conséquent, difficiles à repérer. Au total, 50 de ces 139 détectives ont été interviewés.

Nous souhaitions vérifier certains aspects des théories bien connues de Shearing et Stenning (1982, 1983). Tout d'abord celle du partenaire junior ou de l'associé, théorie voulant que, puisque les policiers ne sont plus à même de tout faire, des agents privés doivent combler les lacunes. La sécurité privée est dès lors traitée comme une adjonction au système de justice pénale. Nous voulions ensuite tester la théorie économique, aussi appelée prévention ou réduction des pertes (loss prevention), théorie selon laquelle les agents privés, employés à contrat, travaillent surtout pour répondre à un besoin économique et privé ${ }^{4}$ vécu par le mandataire.

3. Cette étude peut être comparée à celle de Gill et Hart (1997).

4. Les termes que nous utilisons sont de Shearing et Stenning. Dans le cas contraire, nous indiquons la source. 
Nous ne voulions pas croire qu'un vacuum sécuritaire comblé par la sécurité privée existait en tant que tel. Nous pensions plutôt nous trouver face à l'expansion d'un discours de vacuum sécuritaire. Nous penchions donc pour le modèle de prévention des pertes plutôt que pour une interprétation du phénomène de privatisation en tant que reflet de la complémentarité des forces publiques et des agences privées à des fins d'intérêt public.

Selon nous, les détectives ne se chargeraient pas seulement de travaux de surveillance, mais aussi et surtout du travail d'enquête. Telle était l'hypothèse à la base de la première étude, celle-ci voulant que plus l'État constitutionnel ronge les compétences des forces policières publiques, plus ces dernières ont tendance à faire appel au circuit privé et à ses agents pour se charger, en sous-traitance, du sale boulot souvent illégal (dirty work argument) (Marx, 1987). Cela étant dit, le secteur privé n'hésiterait pas à essayer de tirer profit du réseau des vieux amis (old boys network), en particulier du réseau de policiers qui ont quitté la police pour travailler dans le secteur de la sécurité privée (blue drain), ainsi que des policiers travaillant pour le secteur privé après leurs heures de travail (moonlighting).

Enfin, étant donné qu'il n'existe pas de législation en la matières', se pose également le problème du contrôle de l'État démocratique sur la privatisation.

\section{Les détectives privés de la deuxième génération}

Lors de notre première étude, nous avons constaté qu'une autre catégorie de détectives privés occupait le marché, soit celle des enquêteurs spécialisés. En 1991, nous avons reçu une nouvelle demande d'étude du ministère des Affaires intérieures, cette fois sur l'essor des enquêtes privées spécialisées effectuées par des employés dépendants de compagnies (in house) ou par des contractants. (Boon, 1995a, b). Dans un premier temps, nous avons été amenés à réaliser une étude assez générale sur la présence d'enquêteurs de ce genre au sein de différentes institutions commerciales et financières afin d'avoir une vue d'ensemble sur les matières et les personnes en présence. Finalement, nous avons concentré nos efforts sur le département des enquêtes sur les fraudes au sein des compagnies d'assurance automobile. Nous avons commencé par une discussion préliminaire avec des témoins privilégiés. Par

5. Après l'enquête, fut votée, le 19 juillet 1991, la Loi organisant la profession de détective privé, Moniteur Belge du 2 octobre 1991. La loi fut remaniée le 30 décembre 1996, Moniteur Belge du 14 février 1997. Il s'agissait de formuler les conditions de l'accès à la profession et de l'exécution des tâches limitées ; de prescrire une tenue distinctive ; d'organiser la formation et le contrôle. Par la loi de 1997, la formation et le contrôle deviennent plus stricts. 
la suite, 82 personnes ont été interrogées : 34 membres des forces de police, 23 membres du personnel et 25 autres personnes (détectives privés, enquêteurs sous contrat, avocats, experts, employés de firmes de courtage). L'analyse de quelque 40 dossiers nous a fourni un grand nombre d'informations supplémentaires quant au modus operandi des enquêteurs privés, ainsi que sur leur interaction avec les forces publiques. En outre, afin de mieux comprendre certaines situations, il s'est avéré utile de prévoir une étape d'une semaine d'observation participante des enquêtes privées. Enfin, toutes les compagnies d'assurances opérant sur le marché belge ont reçu un questionnaire concernant l'organisation des enquêtes sur les fraudes.

Sur la base de ce matériel, nous avons pu tester un certain nombre $d$ 'hypothèses issues de notre première recherche et $d$ 'autres recherches faites à l'étranger :

- le travail du détective privé ne devrait pas être très différent de celui de l'enquêteur policier, exception faite du cadre légal d'opération ;

- les investigateurs privés jouissent sans doute d'une plus grande liberté que les enquêteurs de police, notamment parce qu'ils ne sont pas souvent tenus de fournir de preuves légales ;

- les modalités de contrôle de l'État sur les investigateurs privés seraient restreintes ;

- enfin, les relations des investigateurs privés avec la police seraient plus intensives et s'organiseraient principalement autour d'activités d'échange d'informations et de lutte contre le crime (de fraude) proprement dit.

De même, plusieurs questions se posaient : observerions-nous un partenariat entre les sphères publique et privée - ce que l'on appelle aussi dans la littérature une relation symbiotique où deux organismes inégaux coopèrent en vue d'un profit réciproque (Cunningham, Strauchs et Van Meter, 1990) - ou bien constaterait-on la prédominance de l'un ou l'autre de ces secteurs? Le secteur privé tirait-il toujours profit du blue drain et du ola boys network ? La police publique invoquerait-elle le dirty work argument - que nous retrouvons aussi sous l'appellation système hydraulique - voulant que plus les compétences et les moyens des policiers sont étroits ou limités, plus ceux-ci font appel aux agents privés pour se livrer à des activités illégales dans la recherche d'éléments d'enquête (Marx, 1987) ?

Finalement, nous avions déjà quelques indications sur la protection légale des clients des compagnies d'assurances : celle-ci serait déficiente du fait des enquêtes privées, du recours aux systèmes de justice privée et de la non-intervention, pour le moins radicale, tant de la police que de la justice publique (Ocqueteau, 1986, 1990). En outre, on pouvait supposer que les 
agences privées influençaient de façon directe ou indirecte le contentieux pénal en choisissant les cas qui seraient transmis aux autorités judiciaires ${ }^{6}$.

\section{Police privée et respect des droits fondamentaux}

Cette troisième étude fut menée dans le cadre du programme de recherche Le Citoyen et sa protection légale lancé par le Département fédéral des affaires scientifiques, techniques et culturelles entre janvier 1992 et décembre 1994. Suite à l'acceptation de notre projet de recherche, trois chercheurs - deux criminologues-sociologues et un juriste - ont eu l'occasion de se pencher sur les problèmes liés à l'insécurité légale, l'inégalité et le manque de protection juridique du citoyen impliqué dans le cadre de la surveillance, du gardiennage ou des enquêtes privées (Van Laethem, Decorte et Bas, 1995). Lors de nos études précédentes, nous avions été confrontés au problème du (non-) respect des droits fondamentaux de la personne. Une approche interdisciplinaire se basant sur la sociologie et le droit s'avérait nécessaire. Nous avons affiné notre étude en la circonscrivant à quatre domaines d'application :

- les grandes surfaces commerciales faisant appel aux détectives et au gardiennage privés ;

- les méga dancings où les surveillants font office de portiers et de gardes (contrôlant le trafic de drogues, le vandalisme, la violence);

- les stades de football où un personnel d'accueil (les stewards) et des gardiens privés sont employés ;

- les enquêteurs privés, domaine que nous connaissions déjà assez bien?

Comme précédemment, nous avons commencé cette étude par une série d'entretiens avec des témoins privilégiés. Par la suite, parmi les différentes catégories de personnes impliquées, environ 150 personnes furent interviewées. La technique de recherche principale fut toutefois l'observation participante, intensive et de longue durée. Le juriste de l'équipe s'est attelé

6. Au moment de l'enquête était déjà en cuvre La loi du 10 avril 1990 sur les entreprises de surveillance, de gardiennage et les services de sécurité interne. Cette loi définit des conditions d'accès au travail, délimite les tâches de ces agences, tout en stipulant qu'il leur est strictement défendu d'exercer des fonctions policières ou des tâches de détective privé.

7. Les services de sécurité interne qui fonctionnent dans l'enceinte des entreprises restaient en dehors de la recherche. Eux aussi ont été réglementés par la loi du 10 avril 1990. Mais il faut faire remarquer que les stewards dans les stades de football, les inspecteurs dans les supermarchés et les gardiens dans les discothèques fonctionnent très souvent comme de vraies polices d'entreprises, puisqu'ils surveillent très souvent aussi bien le personnel que les clients. 
à une analyse approfondie du système législatif belge, des traités internationaux, des conventions sur la protection de la vie privée et des droits de la personne, ainsi que de la jurisprudence. Par la suite, il a comparé les résultats de l'étude sociologique de terrain aux dispositions légales étudiées afin de rendre compte de la légalité des pratiques observées.

L'étude nous a permis de reprendre un certain nombre d'hypothèses et de les tester, soit :

- l'hypothèse du système symbiotique concernant la genèse d'un partenariat entre le public et le privé, celle du fonctionnement d'un système hydraulique ou théorie du sale boulot, ainsi que celles du blue drain et du moonlighting en tant qu'éléments favorisant le secteur privé ;

- l'idée que peut-être d'autres enjeux que la prévention ou la réduction des pertes mènent l'action de l'agent privé. À l'instar de Hoogenboom (1988a), nous avons tenu à ajouter que les forces publiques se concentrent probablement plus sur les auteurs d'actes criminels, alors que les agences privées s'occupent principalement des intérêts et des droits des victimes, c'est-àdire principalement de personnes ou de compagnies privées ;

- la possibilité que des informations personnalisées soient échangées entre les services de police publique et les agences privées, faisant en sorte que les enquêtes publiques et privées s'entremêlent, rendant par là impossible la détermination des responsabilités ;

- l'éventualité que les surveillants privés et, à plus forte raison, les investigateurs privés, profitent vraisemblablement du vide législatif en la matière. Leurs moyens financiers et techniques leur permettent par ailleurs d'agir de manière passablement équivoque, résolvant de la sorte certains problèmes en empruntant des voies illégales, dans des pratiques justifiées par des fins d'efficacité. Nous pensions alors que la concurrence régnant sur le marché des agences privées ne pouvait qu'exacerber cet état de fait ;

- le fait que des banques de données sont probablement créées sans le moindre contrôle, ce qui pose la question de la protection de la vie privée des citoyens. En termes légaux, le citoyen est protégé contre toutes formes d'abus. Cependant, les mécanismes d'opérationnalisation et de contrôle s'avèrent plutôt inadéquats.

Enfin, les acteurs privés appliquent en bout de ligne une forme de justice privée (Ocqueteau, 1986, 1990). Ils décident souverainement si l'intervention de la justice publique est justifiée ou ne l'est pas. Or, les intérêts commerciaux des agences privées ne sont sans doute pas ceux du système de la justice pénale. Cette justice privée ne garantit ni l'impartialité, ni l'égalité 
face à la loi. Elle ne garantit pas davantage la sécurité et l'assistance légale, la publicité et la motivation des décisions.

\section{Pour terminer : la police grise}

Toujours financés par le même programme de recherche, deux chercheurs - un criminologue-sociologue et un juriste - ont pu poursuivre l'étude de la police grise durant l'année 1995 (Decorte et Van Laethem, 1997). Nous avions décelé ce phénomène à maintes reprises durant nos études précédentes, d'où notre demande d'en faire un objet de recherche explicite (Decorte et Van Laethem, 1997 ; Decorte, Van Laethem et Van Outrive, 1997). La matière principale de cette étude nous a été fournie par le cas d'un officier de la police judiciaire accusé de falsification, d'insubordination, d'abus de pouvoirs et de violation du secret professionnel. Ce cas impliquait 24 autres personnes. Plus intéressant sans doute est le fait que le dossier judiciaire a révélé nombre d'interactions problématiques entre la police et certains acteurs privés : enquêteurs privés, informateurs et hommes d'affaires. Ce genre d'information mettait en perspective une grande variété de contacts entre la sphère publique et la sphère privée. L'étude s'est inspirée de différentes sources : une analyse de la presse ( 10 journaux et 5 hebdomadaires), 70 dossiers judiciaires ( 40000 pages) traités en partie, et les arrêts de la Cour d'appel. Une trentaine d'entretiens non directifs avec des témoins privilégiés de l'affaire ont été effectués. Il s'agissait de policiers, de hauts fonctionnaires du département de la justice, d'enquêteurs privés, d'informateurs, de magistrats et d'avocats. Les chercheurs ont aussi eu recours à l'observation participante et ont enregistré les plaidoiries lors des sessions en cour d'appel. Il va sans dire qu'une critique sérieuse des différentes sources était capitale, et qu'il était nécessaire de procéder par triangulation. Un échantillon opérationnel et raisonné de sept histoires au contenu jugé le plus riche en informations et le plus significatif a été construit afin que nous puissions nous livrer à une analyse structurelle aux plans meso et micro descriptifs.

Ayant déjà acquis, lors des études précédentes, une certaine perception de l'importance des variations de formes et de modalités des interactions entre la sphère publique et privée, nous avons pu, une fois de plus, formuler un certain nombre d'hypothèses :

- l'absence de cadre légal ou les conséquences latentes des règles, qui ont tendance à occulter la réalité de l'interaction entre les sphères publique et privée, aboutiraient à des interactions informelles et problématiques ;

- l'influence croissante des compagnies industrielles, financières et commerciales sur les enquêtes concernant les crimes dont elles sont victimes les mettraient en position dominante face à la lutte contre le crime et les 
violations des lois. Cela provoquerait également une confusion entre leurs intérêts et ceux des forces policières ;

- moins les membres des forces de police seraient socialisés selon les normes en vigueur pendant leur période de formation, plus ils auraient tendance à utiliser des techniques illégales. Une de ces techniques serait précisément l'interaction intensive avec les agents privés (quasi) professionnels et les compagnies. En somme, lorsque l'efficacité dans la lutte contre le crime prime sur la légalité, il y a de fortes chances de voir se multiplier les interactions informelles et problématiques. La genèse d'une certaine culture policière commune sous la forme dite de partenariat junior résulterait d'un mélange des intérêts publics et privés. Ce phénomène serait toujours influencé par le blue drain, le old boys network et le moonlighting ;

- si on laisse aux enquêteurs policiers une autonomie leur permettant de définir des priorités dans le travail d'enquête, une interaction informelle et problématique pourrait se dessiner. Si, d'une part, le contrôle du magistrat sur les activités liées à l'enquête policière diminue et que, d'autre part, l'autonomie des policiers sur le terrain (street level bureaucary) augmente, l'interaction entre les sphères publique et privée pourrait devenir encore plus informelle et plus problématique ;

- L'interaction entre les sphères publique et privée reflète la fameuse relation symbiotique, car les deux parties apparaissent complémentaires et s'échangent des informations pour obtenir leurs propres résultats. Mais les deux organisations ne feraient pas plus usage de leur autonomie qu'il n'est nécessaire pour obtenir les résultats attendus. Les restrictions réelles ou perçues des moyens, des compétences, des canaux d'information et du savoir-faire des uns et des autres réduiraient les chances de pouvoir exploiter cette autonomie. Le simple fait que l'argument du sale boulot ou du système hydraulique et des soi-disant banques de faveurs ${ }^{8}$ soient évoqués renforce cette hypothèse ;

- finalement, l'essence même de la notion de police grise se résumerait en une forme de collaboration. " où le fait de savoir qui fait quoi et qui est responsable de quoi »n'est plus tellement clair (Hoogenboom 1988b).

\section{LES RÉSULTATS CONFRONTÉS AUX THÉORIES EN VOGUE}

\section{Qu'en est-il de la fameuse théorie du partenaire junior ?}

Cette ancienne théorie postule qu'il y aurait un vacuum sécuritaire laissé par la police et que ce vacuum serait comblé par des agents de sécurité privée.

8. Hoogenboom (1994) a développé cette idée d'une banque de faveurs voulant que chaque partie investisse dans un système d'échange de services. On peut dès lors se demander qui y apporte le moins tout en en retirant le plus. 
Nous avons constaté que cette théorie tient davantage du discours que d'une réalité vérifiable. Les enquêteurs privés de la première génération - les détectives privés polyvalents - tout comme les agents de gardiennage et de surveillance adaptent leur discours à cette théorie et l'utilisent afin de justifier leur situation professionnelle. Ces agents renvoient à la dite théorie pour expliquer les raisons pour lesquelles leurs clients s'adressent à eux. Cet argument s'avère utile lorsqu'il est question de justifier leur existence par rapport aux autorités ou aux forces de police. En revanche, on constate que, dans la pratique, les détectives privés n'apparaissent que très rarement comme de véritables agents de lutte contre le crime, puisqu'ils passent le plus clair de leur temps à récolter des informations de toutes sortes. Vu sous cet angle, on observe très rarement une complémentarité dans les activités des uns et des autres. Toutefois, lorsqu'ils sont impliqués dans la lutte contre le crime, les agents de sécurité privée utilisent les mêmes moyens que les forces publiques de l'ordre, sans toutefois en avoir les compétences. Cette dernière situation est plus souvent celle des agents de surveillance et de gardiennage quand, en pratique, ils jouent au policier. Mais nous devons constater que l'on ne trouve pas de traces de ce soi-disant décalage entre le discours et la pratique lorsqu'on observe la situation d'autres agents privés plus spécialisés, ou de la deuxième génération : ceux-ci copient tout simplement le travail policier et s'occupent des crimes, surtout des fraudes diverses. Il en est de même quand il s'agit de l'interaction entre les policiers et toutes sortes d'informateurs, comme nous l'avons constaté dans la dernière recherche. En somme, cette théorie est assez floue et superficielle et ne permet pas toujours de rendre compte de la réalité des phénomènes.

\section{Les relations privé-public sont-elles symbiotiques ou parasitaires ?}

Cette question avait déjà surgi lors de la deuxième enquête, mais ce sont surtout la troisième et la quatrième études (en particulier) qui donnent des réponses.

Si l'on parle d'interactions entre sphères publique et privée, c'est tout d'abord parce qu'il ne s'agit pas forcément de coopération, terme renvoyant à des acceptions souvent très différentes. Nous avons aussi découvert que les relations ne sont pas toujours réciproques, ni même équilibrées entre les sphères privée et publique. Beaucoup d'interactions sont asymétriques et même parasitaires, tout d'abord parce qu'elles ne prennent pas toujours le consensus comme base et peuvent parfois être imposées. Il ne s'agit donc pas toujours d'un prêté pour un rendu. En effet, si l'on met de côtê les interactions temporaires entre les policiers et les agents privés pour se concentrer sur les interactions prolongées et plus systématiques, l'analyse révèle un tout autre tableau que celui des relations symbiotiques. L'interaction n'est plus 
équilibrée, elle devient tout à fait parasitaire. Elle met à jour une relation entre deux organisations inégales qui ne collaborent plus dans la perspective d'un profit mutuel, puisque l'une d'elles vit au crochet de l'autre et l'influence de façon négative. Pour arriver à ses fins, une organisation abuse de l'autre, sans se soucier des buts de cette dernière. Dans cette relation, le principe du donnant-donnant peut prendre plusieurs formes et n'est nullement limité à l'échange d'information. Il inclut : échanges de savoir et de moyens techniques, dons, prêts personnels, transferts de cas judiciaires, etc. Cette situation est parfois dangereuse pour les officiers de police, parce qu'elle peut leur faire quitter le domaine de la légalité et les rendre tributaires, pour ne pas dire dépendants, des acteurs privés. On a pu voir que les banques de faveurs ainsi constituées permettent un jeu de pouvoir et une sorte de stratégie commerciale à profit inégal.

\section{Qu'en est-il du fameux système hydraulique ou de l'argument dit du sale boulot?}

Dès la première enquête, nous en avons trouvé des traces, mais le fonctionnement d'un tel système est devenu tout à fait manifeste au cours de la dernière recherche.

À ce propos, il est essentiel de connaître la différence de cadre légal déterminant les activités de la police et celui des acteurs privés. Les compétences de la police en matière d'investigation sont formellement restreintes. Nous avons constaté que la police laissait de temps à autre le sale boulot aux agences et acteurs privés. On peut parler à cet égard d'une sorte de soustraitance. Il est parfois sous-entendu qu'en raison de ses compétences trop limitées, la police se voit obligée de demander l'intervention des agents privés pour exécuter certaines tâches qui ne lui sont pas permises.

Nous avons confirmé notre hypothèse selon laquelle la police belge ne rencontre pas d'obstacles ou de contrôle dans son utilisation des instruments traditionnels d'investigation et qu'elle jouit dans ce domaine d'une autonomie assez (voire trop) large. Mais notre dernière enquête sur la police grise nous a permis de constater qu'elle est plutôt confrontée à un retard dans la formation et à un manque de moyens. En effet, i] s'avère que la police belge ne parvient pas à faire face à de nouvelles formes de criminalité, telles la criminalité financière, économique ou organisée. La police fait alors appel au secteur privé pour une forme de coopération qui n'est pas nécessairement illégale et qui se révèle fort utile.

\section{La police perdante, le privé gagnant?}

La première et la troisième enquêtes nous laissaient penser que les profits de la collaboration étaient plutôt réciproques. Les résultats de la deuxième 
recherche nous donnent toutefois une autre image : les compagnies d'assurances profitent largement du travail policier. La dernière recherche confirme assez nettement cette image : les policiers sont finalement victimes des agents et agences privées.

Examinons tout d'abord la situation des membres de la police publique. Nous nous sommes rendu compte que, dans certaines conditions, leur situation pouvait devenir extrêmement pénible. C'est le cas notamment :

- lorsqu'ils jouissent d'une trop grande latitude dans la définition de leurs priorités de travail ;

- lorsque les contrôles internes ou certaines formes de contrôles externes sont trop laxistes, voire inexistants. Ils font alors ce qu'ils veulent dans leur travail journalier ;

- lorsqu'une forme de concurrence règne entre les différents services de police ou au sein d'un même service. Ces conditions produisent une escalade dans la chasse aux bons points. À l'évidence, les interactions informelles et problématiques sont facilitées par cette course aux résultats et aux points, de même que l'esprit de concurrence qui règne entre les policiers d'un même service de police ou entre les services de polices euxmêmes ;

- lorsqu'ils doivent faire appel aux informateurs et doivent trouver des fonds pour les rendre bavards ;

- lorsqu'ils doivent demander aux acteurs privés de se charger du sale boulot ;

- lorsque la police grise sous-entend que l'on fasse appel à un échange illégal d'information en raison de l'existence de zones de frictions entre efficacité et légalité.

D'autre part, le rôle des agents et des compagnies privées ou des informateurs notoires de la police est plus important qu'on ne le pense. Tous ces agents ou agences disposent de canaux d'information bien développés et d'importants moyens financiers, économiques et techniques. Cela leur permet de peser de tout leur poids sur le travail policier. Ils peuvent donc avoir une emprise sur les enquêtes concernant les crimes dans lesquels ils sont impliqués en tant que victimes ou sur celles où ils risquent d'éventuelles pertes. Ils peuvent donc décider de la marche à suivre. Ainsi, ils peuvent évaluer si le cas vaut la peine d'être investigué ou s'il est préférable de l'abandonner à la police et au système pénal ou au système de justice civile. Par conséquent, ils décident de ce qu'il adviendra des résultats de l'enquête. Nous avons en effet constaté que l'agent (ou l'agence) privé faisait à la fois figure 
d'enquêteur et de procureur, tandis que l'employeur ou le commanditaire, comme partie intéressée, était en même temps juge et partie. Notons qu'un traitement discrétionnaire est dès lors toujours possible. Faut-il rappeler que ces éléments entravent l'application de la justice et provoquent la confusion entre les intérêts privés et publics ?

\section{Les phénomènes du old boys network, du blue drain et du moonlighting moins présents que dans d'autres études faites à l'étranger"}

Ce sont surtout les deux premières enquêtes qui nous ont révélé ce fait. Cependant, lorsque ces phénomènes se présentent, ils tendent à faciliter l'interaction et même la coopération entre secteurs public et privé. Il semble que les contacts entre les membres de la police et les acteurs privés ayant derrière eux une carrière policière sont moins souples qu'on ne le pense et que ces gens se témoignent une méfiance réciproque.

Nous n'avons par ailleurs pas seulement été confrontés au phénomène du moonlighting, mais aussi à celui que nous avons nommé sunlighting, soit des policiers qui se mettent au service des acteurs privés ou des agences privées, non seulement après, mais aussi pendant leurs heures de travail. Jusqu'à présent, nous n'avons pas été en mesure de définir ce que recouvre la notion de culture policière commune (Euler, 1980). S'agit-il d'un langage commun ? D'un mode d'opération commun? Nous ne savons pas non plus si cette culture s'installe plus rapidement lorsqu'il s'agit d'une coopération entre agents privés et anciens policiers.

\section{Y-a-t-il un complexe police-industrie ?}

O'Toole (1978), Marx (1987) et Hoogenboom (1994) suggèrent qu'un complexe police-industrie est en pleine expansion. Nos études ont révélé que ce genre de réseau formel est plutôt l'exception que la règle, et que le caractère prédominant de ces interactions est nettement interpersonnel et informel. Les policiers qui travaillent sur le terrain (les soi-disant street level bureaucrats) jouissent d'une marge de liberté considérable et ceci malgré les compétences formelles assez réglementées et limitées qui sont les leurs. Par conséquent, les intérêts personnels ont souvent tendance à prendre le pas sur ceux de l'organisation à laquelle les individus appartiennent. Il n'était donc pas aisé pour nous de savoir clairement dans quelle mesure la distinction entre privé et public est en train de disparaître, aussi bien sous l'influence d'une légitimation accrue du secteur privé par l'État, que sous celle d'une plus forte professionnalisation de ce secteur, ce qui pourrait avoir pour effet

9. En particulier Bunyan (1977), O’Toole (1978) et Reiss (1988). 
de brouiller les frontières entre les missions et fonctions de police (Ocqueteau, 1990).

Il faudra voir, dans l'avenir, si les autorités publiques et les agences privées ne vont pas conclure des accords octroyant aux entreprises privées des tâches de surveillance et de gardiennage dans des espaces, bâtiments ou institutions publics. Mais lors de nos enquêtes, nous n'avons pas encore trouvé de tels indices.

\section{Qu'en est-il finalement de la théorie de la prévention des pertes ?}

La théorie traditionnelle s'enracine, dans une large mesure, dans la notion de prévention ou réduction des pertes (le policing for profit de Shearing et Stenning, 1982). À travers les résultats de toutes nos recherches, nous nous sommes rendu compte que cette notion était trop étroite et devait par conséquent être remplacée par celle de l'intérêt du client ou de l'instance mandante. Cette dernière notion a également une dimension économique, mais son sens est élargie : elle inclut les suites d'une expérience négative des compagnies avec les forces de police, la conviction que le secteur privé peut être plus efficace et la volonté de garder un contrôle sur l'enquête, c'est-à-dire de maîtriser l'information et d'éviter un certain type de publicité.

Nous avons également constaté d'importantes divergences d'intérêts entre le client ou les instances mandantes et leur mandataire-investigateur. Les clients ou commanditaires ont des intérêts qui, d'un côté, dépassent la simple prévention des pertes et, d'un autre côté, ne rencontrent pas nécessairement les objectifs de l'investigateur (par exemple, la lutte contre le crime). Ceci est apparu clairement dans notre deuxième recherche auprès de la seconde génération d'enquêteurs, et lors des observations effectuées pour notre troisième recherche menée auprès des agents de surveillance qui entendaient copier les modes d'action policiers. Cela s'est révélé de façon bien plus nette qu'au sein de la catégorie des détectives privés polyvalents de la première génération, objet de notre enquête initiale. Quant à notre dernière recherche sur la police grise, elle nous a révélé à ce sujet une situation plutôt anomique et confuse : lutte anti-crime, gain pécuniaire et prévention des pertes s'entremêlaient constamment. Nous avons également constaté que, dans le secteur de la sécurité privée, la notion de déviance renvoie à autre chose que la notion de criminalité traditionnelle. Tantôt, elle couvre un vaste champ sémantique - des fautes morales ou des négligences - tantôt elle renvoie à des notions plus restreintes, comme le fait de ne pas tenir compte de certains vols d'objets ordinaires ou de l'abus des moyens de communication. La notion de déviance comporterait également tout ce qui entrave la continuité de la production ou de la prestation du service. On est alors loin du calcul des simples pertes matérielles. Le rôle de l'investigateur et sa 
proactivité ne sont plus limités à l'empêchement de ces pertes (Shearing et Stenning, 1982 ; Ocqueteau, 1990). En fait, la mission renvoie à une proactivité conçue sans limites véritables.

\section{Des situations de marché différentes ?}

Lors des deux premières enquêtes, nous avons constaté des différences entre les profils professionnels de deux catégories d'enquêteurs privés. Nous avons fait usage du concept de génération tout en ayant recours à une approche empruntée à la sociologie du travail qui définit la distinction entre le marché du travail primaire et secondaire. La première génération d'agents de sécurité privée occupe le marché secondaire : il s'agit de personnes généralement non qualifiées, qui ont répondu aux annonces publicitaires de recrutement. Leur position est instable, ils sont en somme dans une situation professionnelle peu enviable. Nous avons ensuite constaté que ce marché est relativement cloisonné : on y trouve d'une part des détectives généralistes offrant toute une gamme de services en tant qu'investigateurs polyvalents ${ }^{(1)}$, et d'autre part de simples agents de gardiennage et de surveillance. Seul un nombre limité d'entre eux se spécialisent et peuvent voir évoluer leur position sur le marché du travail (principalement la catégorie des détectives privés). La seconde génération occupe le marché primaire. Il s'agit d'agents qualifiés et spécialisés qui ne font pas appel aux annonces. Leur emploi est stable et rémunérateur. Ils travaillent comme salariés pour une compagnie ou comme indépendants à contrat. Dans tous les cas, ils effectuent des enquêtes spécialisées.

\section{Les activités des agents privés portent-elles atteinte aux droits fondamentaux?}

On néglige souvent les implications, pour le citoyen, du fonctionnement des agents de surveillance et de gardiennage ainsi que de la police grise. Nous avons constaté des inégalités dans la façon dont les citoyens sont traités et relevé à ieur égard une absence de garantie de se voir assurer leur sécurité. Les enquêteurs ou surveillants privés font peu de cas du respect des lois, non seulement lorsqu'elles ont trait à la protection de la vie privée et aux libertés individuelles, mais aussi lorsqu'elles portent sur la liberté de réunion ou d'association. Il en est de même pour les garanties d'accès à la justice et le droit à un procès équitable. En définitive, ce sont les valeurs fondamentales de l'État constitutionnel qui ne sont pas respectées. La priorité étant donnée à la satisfaction des clients ou mandataires, le respect de la protection des

10. Selon un détective privé : « Quand on ne sait plus quoi faire en Belgique on devient détective ». 
libertés ne participe aucunement aux objectifs poursuivis. Nous avons en effet noté que les notions de vie privée et de libertés individuelles semblent en voie d'érosion dès qu'une enquête privée est en cours, et que très souvent l'efficacité prime sur la légalité des moyens employés aux fins d'enquête. L'industrie de la sécurité privée ne contribue pas vraiment à combattre le crime ou à assurer la sécurité des gens. D'autres intérêts s'imposent : l'intérêt propre de l'organisation ou de la compagnie et même les intérêts personnels des agents privés.

Nous avons ainsi identifié le risque d'apparition d'une sécurité de classe (au départ d'une enquête de classe) où la protection et la récupération des pertes seraient réservées à ceux qui seraient en mesure de le faire et de se défendre. Nous avons aussi mis à jour la victimisation de clients de compagnies d'assurance automobile, de clients de supermarchés et de certaines catégories de visiteurs des grands espaces, de clients de couleur des discothèques, et de certains supporters dans les stades de football. Un constat qui renvoie à l'idée de rupture de la solidarité sociale.

Il devient de plus en plus difficile pour le citoyen d'identifier qui est censé se charger de telle ou telle tâche policière et de savoir vers qui se tourner préférablement. L'existence d'une telle zone d'ombre rend malaisée la distinction entre pratiques légales et illégales. Sur le terrain, on ne parvient plus à attribuer clairement la responsabilité de telle ou telle pratique à l'un ou l'autre agent compétent. Cependant, nos hypothèses n'ont pas été confirmées dans leur ensemble. De fait, il n'y a pas, à proprement parler, de lacunes en matière législative, pas de vacuum légal : il existe une législation sur la protection de la vie privée et des libertés individuelles et des lois qui réglementent les activitês des détectives privés et des agents de surveillance ou de gardiennage. La possibilité de prendre des sanctions à leur endroit existe bel et bien. Le problème réside plutôt dans le contrôle insuffisant de l'application de la loi et dans l'ignorance des citoyens quant à leurs droits. En outre, comment savoir si l'on est l'objet d'une mise sous surveillance ou d'une enquête privée? Le citoyen moyen n'a pas les ressources financières ou n'a tout simplement pas l'audace d'entreprendre les démarches nécessaires pour attirer l'attention des pouvoirs publics sur certaines pratiques illégales. Il est nécessaire d'organiser un meilleur accès aux bureaux et aux services des ombudsmans, afin que les gens puissent aller s'y plaindre de certaines bavures commises par des agents privés. Il est également indispensable de pallier le manque d'information que l'on connaît actuellement par la publicité des incidents et de la situation légale. Il faut aussi prévoir des moyens de contrôler plus efficacement les agents privés et penser à améliorer leur formation.

Par ailleurs, on pourrait affiner les législations existantes : les policiers ont besoin d'une définition plus claire de ce que l'on entend par preuves 
illégales fournies par une tierce personne. Enfin, une réglementation légale de l'emploi des méthodes d'investigation spéciales et nouvelles, ainsi que de l'usage des informateurs est aujourd'hui devenue essentielle.

\section{Et le contrôle étatique?}

Policing, cette notion fourre-tout ne fait manifestement plus partie du terrain de chasse exclusif de l'État. Exercer un contrôle officiel dans nos sociétés est une question compliquée qui suppose la spécialisation et la professionnalisation des activités.

Après avoir analysé tous ces résultats de recherche, nous nous sommes demandés s'il ne serait pas opportun de réserver à l'État un contrôle assez strict des activités de sécurité privée. En ce qui concerne le métier de détective privé, nous avons plaidé pour une organisation et un contrôle assez sévère de la profession, ainsi que pour une application efficace de la législation sur la protection de la vie privée (en raison notamment du fait que la clientèle des investigateurs privés est composée pour les trois quarts de personnes privées et pour un quart seulement de compagnies). Nous avons également conseillé d'appliquer pleinement la Loi sur les détectives privés aux détectives de la deuxième génération. Après avoir constaté que des gardiens et surveillants privés opéraient régulièrement sans licence ou travaillaient comme des détectives privés, voire comme des policiers, nous avons été amenés à insister davantage sur la nécessité d'intensifier le contrôle par une application plus stricte des lois, un meilleur respect de la légalité et des droits des citoyens ainsi que par l'assurance d'un traitement équitable. Ceci, non seulement au travers des textes légaux, mais aussi, le plus souvent possible, dans la pratique, afin de réellement éviter les abus et de les sanctionner quand ils se produisent. Nous avons dû également insister sur le devoir d'information des autorités auprès des citoyens concernant leurs droits.

Lors de toutes nos enquêtes, et principalement celle concernant la police grise, nous avons constaté combien l'absence de législation régissant l'usage des techniques spéciales d'investigation et le recours aux informateurs pouvait être dommageable. La police publique manque d'information et n'est pas formée à respecter scrupuleusement les droits de la personne, pas plus qu'à composer avec toutes sortes d'agents et d'agences privées. Nous avons également constaté que la guerre des polices était très néfaste et que le contrôle de la magistrature sur la police, tout comme sur la police grise, laissait à désirer. Quant au monde politique, ne devrait-il pas se tenir au courant de ce qui se passe en matière de sécurité et prendre les mesures de contrôle et de surveillance qui s'imposent ? N'est-il pas raisonnable d'affirmer que l'attitude libérale du laissez faire, laissez passer encourage l'illégalité et le manque de respect des droits de la personne, créant ainsi un sentiment 
croissant d'inégalité entre les catégories et les groupes de citoyens ? Tout cela n'a-t-il pas pour effet de porter atteinte aux fondements de la solidarité sociale ? Cela ne conduit-il pas à une inégalité en matière de sécurité et de protection? Si l'évolution qui s'opère dans le domaine des agences privées de sécurité n'échappe pas tout à fait à l'attention des autorités publiques, il semble toutefois que les pratiques policières privées n'aient pas eu droit aux soins et à l'attention qu'elles méritent. Nous avons aussi l'impression que, dans certains pays, les autorités ont recours à la politique de l'autruche. $\mathrm{Ce}$ secteur étant un des rares à créer de nouveaux emplois, on a parfois le sentiment qu'il est ainsi, en quelque sorte, protégé.

D'autre part, les pouvoirs publics, dans les sociétés à État fort, se sont vus contraints de réagir aux défis posés par la gestion commerciale de la sécurité, sans avoir pris l'exacte mesure de toutes les implications du phénomène ... (il y a) une sous-estimation publique des caractéristiques et des enjeux réels que pose la sécurité privée (Ocqueteau, 1990 : 97).

Au cours de ces dernières années, les autorités belges semblent avoir fait un effort en tentant de contrôler le secteur de la sécurité privée. Nous avons cependant quelques indications qui tendent à montrer qu'elles souhaitent déléguer ou, en d'autres termes, qu'elles entendent responsabiliser ce secteur. Mais est-ce bien, comme on le prétend, pour des raisons budgétaires ? Une autre explication, fondamentalement politique, réside peut-être dans le fait que les représentants de l'autorité étatique ne peuvent pas tout assumer seuls et doivent déléguer ou sous-traiter avec des spécialistes. Il serait donc possible que le contrôle suprême de l'État devienne plus indirect, mais qu'une de ses tâches principales reste tout de même d'assumer la responsabilité ultime de la loi et de l'ordre, sous le contrôle du Parlement.

Il nous semble cependant qu'il y a beaucoup de raisons pour lesquelles l'État ne légalise pas tout simplement, mais suit et contrôle aussi lucidement et efficacement que possible le phénomène de la privatisation d'un certain nombre de tâches qui traditionnellement étaient exercées par la police publique. L'État garde en effet un œil sur tous les nouveaux services appelés de sécurité et de justice privée qui se développent, souvent avec beaucoup de professionnalisme, dans l'enceinte des agences et espaces privés ou semiprivés. La gestion de l'ordre et de la légalité est d'intérêt public et ne se réduit pas à un produit commercial, au risque de produire une inégalité de bien-être dans nos sociétés — une sécurité inégale - et de voir se dégrader davantage les relations de confiance entre le citoyen et les autorités publiques. Il serait envisageable pour les autorités publiques de restituer aux citoyens la capacité de traiter certaines situations problématiques. Cependant, cela suppose une plus grande inventivité de la part des autorités publiques afin de trouver de nouvelles formes de contrôle. On pourrait penser, par exemple, 
à « une commission mixte de supervision et de conseil à laquelle peuvent participer des fonctionnaires, policiers et autres, des syndicats, des associations de consommateurs, et qui fait rapport au ministre responsable et au Parlement » (Van Outrive, 1990). De plus, il ne faut pas sous-estimer les changements apportés par le développement de la sécurité privée aux fonctions de la police publique, ainsi qu'au réaménagement de la gestion de la criminalité.

\section{PERSPECTIVES DE RECHERCHES}

Il est certain qu'en Belgique, tout comme dans d'autres pays d'Europe, le développement du phénomène de la sécurité privée est si important qu'il est nécessaire d'assurer une certaine continuité de la recherche dans ce domaine. Celle-ci présente encore beaucoup de lacunes en Belgique et un développement des théories plus globales du contrôle social est à souhaiter.

Entre-temps, nous avons entamé une nouvelle recherche sur les consultants en matière de sécurité. À vrai dire, on ne connaît que très peu de choses sur ce nouveau domaine du marché sécuritaire. Néanmoins, l'information dont nous disposions, ainsi que notre expérience en matière de recherche, nous a permis d'avancer de nouveau quelques hypothèses. Ainsi, nous pensons découvrir des liens entre les consultants et les compagnies d'assurances et de sécurité. L'influence des consultants pourrait faire croitre les demandes d'indemnités, dans la mesure où les compagnies sont encouragées à développer de plus en plus leurs systèmes de sécurité, ainsi que la sécurité des membres du personnel. Les conseillers sont probablement porteurs d'une conception assez spécifique de la criminalité ou de la déviance, située dans la lignée d'une démarche économique, commerciale et technique. Il serait intéressant de tester, encore une fois, l'hypothèse de la recherche de la réduction des pertes comme principale motivation à l'action de la sécurité privée, et d'analyser ce que l'on entend par politique de sécurité et risque. On peut également s'interroger sur l'expertise professionnelle des consultants, vu le manque de programmes spécifiques de formation ou de savoirfaire spécialisé.

Les activités dans le domaine de la sécurité privée, les fonctions, les agences se développent considérablement et attirent toujours de nouvelles clientèles. On peut aussi bien invoquer la théorie très mercantile de la dissuasion par le durcissement des cibles (Mac Mahon, 1996) qu'y voir une valorisation croissante des techniques de surveillance à distance ou de protection. Techniques qui risquent d'ailleurs de supplanter les tâches des agents privés de surveillance assez vulnérables et souvent devenus inutiles. Tout en mettant encore plus en danger la protection de la vie privée et des 
libertés individuelles. Que deviennent entre-temps les tâches des policiers, des vigiles, des fonctionnaires ayant une compétence policière, des policiers administratifs ou des divers types d'inspecteurs? Peuvent-ils garder une certaine efficacité ? Disposeront-ils aussi de la nouvelle technologie ? De qui et de quoi dépendront-ils pour réagir à une certaine criminalité de plus en plus sophistiquée ? La nouvelle conception de police communautaire leur permettra-t-elle de se redéployer et de se repositionner?

Les compagnies de sécurité privée fonctionnent de façon de plus en plus corporatiste : elles s'organisent et se professionnalisent, développent une déontologie. Il y a d'ailleurs beaucoup de mouvement dans le secteur : des fusions, des ventes, de l'internationalisation, de la concurrence exacerbée pour l'obtention des marchés. Jusqu'à présent, le rôle et la position des compagnies d'assurances dans ce secteur, leurs efforts dans le but de socialiser des mécanismes assuranciels ne sont pas très manifestes en Belgique (Ocqueteau, 1997). Nous avons peu d'informations concernant la relation entre les capacités financières des compagnies ou agents privés et la fréquence des interactions financières entre les sphères publique et privée. À combien se monte le budget de ces compagnies pour les enquêtes privées ? Comment, pourquoi et jusqu'où font-elles appel aux autorités publiques pour les aider à résoudre leurs problèmes, tout en y investissant des moyens financiers et technologiques ? Quels sont les dysfonctionnements qui surgissent à cette occasion?

Alors même que l'État entend surveiller davantage ce qui se passe dans ce domaine, on ne connaît pas l'efficacité de son dispositif de contrôle. L'État se donne-t-il vraiment les moyens de rendre ce dispositif effectif ? Quel serait, par exemple, l'impact d'une interdiction de travailler dans une agence privée pour toute personne ayant quitté les forces de police depuis moins de cinq ans? Quelle politique de gestion voit-on se dessiner? Quelles sont les conséquences, pour la gestion de la sécurité, de la privatisation d'un certain nombre de services publics, comme le téléphone, l'électricité, le secteur des banques et des crédits?

L'existence de réseaux plus formels entre la police publique et le secteur privé pourrait constituer un autre sujet de recherche. Des contrats et des relations entre ces deux instances sont en train de se développer dans le domaine des transports publics et du convoyage de fonds et de valeurs, mais aussi dans des lieux comme les ports et les aéroports, les campus universitaires et les stades de football. Des lois récentes sur le blanchiment de l'argent obligent et formalisent certaines interactions, voire la coopération avec la police et les autorités. S'agit-il là d'indices d'un vrai partenariat ? Est-ce que la prescription de telles interactions conduit à des connections latentes et inattendues entre les deux parties ? Les limites existant entre le privé et le 
public ne vont-elles pas s'amenuiser davantage ? Va-t-on vers « une multiplication des entités organisées gérant des ordres plus ou moins autonomes... » ? (Ocqueteau, 1997).

Nous n'avons, jusqu'à présent, pas encore épuisé nos ressources d'information sur l'interaction quotidienne informelle existant entre les secteurs privé et public dans le cadre de la lutte et de la prévention du crime. Nous sommes convaincu qu'il existe une quantité d'habitudes et de pratiques informelles que nous n' avons pas encore étudiées. Ainsi, par exemple, il serait regrettable de négliger l'influence de l'arrivée des nouvelles technologies. Nous pensons qu'il serait intéressant d'étudier, par le biais de l'observation participante, la culture commune existant entre les forces publiques et les acteurs privés qui collaborent de façon de plus en plus systématique.

Les relations entre la magistrature et la police devraient aussi être analysées avec plus de profondeur et rapportées aux interactions entre secteurs public et privé. Pourquoi les magistrats, bien qu'au courant de certaines pratiques intolérables, semblent-ils préférer ne pas réagir ?

Nous ne savons quasiment rien des interactions se déroulant entre la police belge et les services secrets (ou autres...) étrangers résidant dans notre pays. Un autre travail de recherche pourrait nous informer plus précisément sur ce que certaines personnes interviewées ont appelé l'américanisation $d u$ travail de police. Et que penser de l'influence de la germanisation de la police européenne?

Enfin, une attention particulière est à apporter à l'européanisation et l'internationalisation de la sécurité privée, mais aussi aux échanges d'information ayant cours entre les polices publiques et les agents et compagnies privées, échanges eux aussi en voie d'internationalisation. À cet égard, l'absence de contrôle démocratique sur cette police grise internationale est à souligner. Quelle est l'Europe de la sécurité privée ? Par exemple, des professionnels comme les assureurs sont-ils vraiment des acteurs décisifs du marché de la protection ? (Ocqueteau, 1997).

On le voit bien, les questions sont loin d'être épuisées.

\section{RÉFÉRENCES}

ALBARELLO, L., DIGNEFFE, F., HIERNAUX, J.-P., MAROY, C., RUQUOY, D. et de SAINT GEORGES, P. (1995), Pratiques et méthodes de recherche en sciences sociales, Paris, Armand Colin. 
BOON, K. (1995a), De gespecialiseerde private opsporing. Een tip van de sluir opgelicht (Les enquêtes spécialisées privées ; le coin du voile soulevé), Bruxelles, Politeia.

BOON, K. (1995b), «Les acteurs de l'enquête privée des compagnies d'assurances », Déviance et société, vol. 19, n" 2, p. 169-184.

BUNYAN, T. (1977), The History and Practice of the Political police in Britain, London, Quartet Books.

CAPPELLE, J. (1990), Detectives onder de loep (Les détectives examinés à la loupe), Bruges, Politeia Vanden Broele.

CUNNINGHAM, W.C., STRAUCHS, J. et VAN METER, C. (1990), Private Security Trends 1970 to 2000, The Hallcrest Report //, États-Unis, Butterworth-Heinemann.

DECORTE, T. et VAN LAETHEM, W. (1997), Grijze politie, Verklaringen voor publiekprivate interacties in de zaak-Reyniers (Grey policing. Explication de l'interaction de la sphère publique et privée dans l'affaire Reyniers), Bruxelles, Politeia.

DECORTE, T., VAN LAETHEM, W, et VAN OUTRIVE, L. (1997), « La police grise en Belgique : Résultats d'une recherche ", Déviance et société, vol. 21, n"4, p. 365-382.

EULER, S. (1980), "Private security and the exclusionary rule », Harvard Civil RightsCivil Liberties Review, vol. 5, p. 668.

GILL, M. et HART, J. (1997), «Policing as a business : the structure of private investigation », Policing and Society, vol. 7, p. 117-141.

HOOGENBOOM, A.B. (1988a), Particuliere recherche: een verkenning van enige ontwikkelingen (Enquête privée : travail d'expioration sur certains développements), Den Haag, W.O.D.C., Ministerie van Justitie.

HOOGENBOOM, A.B. (1988b), Privatisering van de veiligheidszorg. Feiten en visies (Privatisation de la sécurité. Faits et visions), Stichting Burgerschapskunde, Leiden, Nederlands Centrum voor Politieke Vorming.

HOOGENBOOM, A.B. (1994), Het Politiecomplex, Anvers, Kluwer Rechtswetenschappen.

MAC MAHON, M. (1996), La répression comme entreprise : quelques tendances récentes en matière de privatisation et de justice criminelle, Déviance et société, vol. $20, \mathrm{n}^{\circ} 2$, p.103-118.

MARX, G.T. (1988), «The interweaving of public and private police in undercover work " in SHEARING, C. et STENNING, P. (dir.), Private Policing, (p. 172-186), Newbury Park, California, Sage.

OCQUETEAU, F. (1986), « Police(s) privée(s), sécurité privée : Nouveaux enjeux de l'ordre et du contrôle social », Déviance et société, vol. 10, n 3, p. 259-267.

OCQUETEAU, F. (1990), L'État face au commerce de la sécurité, Paris, P.U.F.

OCQUETEAU, F. (1997), Les défis de la sécurité privée - protection et surveillance dans la France d'aujourd' hui, Paris, L'Harmattan.

O'TOOLE, G. (1978), The Private Sector. Private Spies, Rent-a-Cop, and Police-Industrial Complex, New York, Norton.

PIRES, A., (1997), « Échantillonnage et recherche qualitative : essais théorique et méthodologique » in DESLAURIERS, J. P., GROULX, L. H., LAPERRIERE, A., MAYER, R., PIRES, A. et POUPART, J. (dir.), La Recherche qualitative : questions et pratiques méthodologiques, (p. 150-176), Montréal, Gaétan Morin.

REISS, A. J. (1988), Private Employment of Public Police, study prepared for the U.S. National Institute of Justice, Washington D.C., Printing Office. 
SHEARING, C. et STENNING, P. (1982), Private Security and Private Justice. The Challenge of the 80's, Montréal, Institute for research on public policy.

SHEARING, C. et STENNING, P. (1983), Private Security : Implications for Social Control, Social Problems, vol. 30, n ${ }^{\circ}$ 5, p. 493-506.

SHEARING, C. (1992), The relation between public and private policing, in TONRY, M. et MORRIS, N. (dir.), Modern Policing, (p. 339-434), Chicago, The University of Chicago Press.

SWANBORN, P. (1984), Methoden van sociaal-wetenschappelijk onderzoek (Méthodes de recherche socio-scientifique), Meppel, Boom.

VAN LAETHEM, W., DECORTE, T. et BAS, R. (1995), Private politiezorg en Grondrechten (Police privée et droits fondamentaux), Louvain, University Press.

VAN OUTRIVE, L. (1988), «Une réglementation belge du secteur du gardiennage et de sécurité : question de (dé)légitimation », Déviance et société, vol. 12, $\mathrm{n}^{\circ} 4, \mathrm{p}$. 401408.

VAN OUTRIVE, L. (1990), Privé-politie en/of overheidspolitie : naar een nieuwe privatepublieke orde? (Police privée et/ou police publique : vers un nouvel ordre privépublic ?) in ELIAERTS, C., ENHUS, E. et SENDEN, R. (dir.), Politie in beweging - Bijdrage tor de discussie over de politie van morgen (Police en mouvement Contribution à la discussion sur la police de demain), (p. 221-231), Arnhem, Kluwer, Anvers, Gouida Quint. 